\title{
MONIC AND MONIC FREE IDEALS IN A POLYNOMIAL SEMIRING
}

\author{
LOUIS DALE
}

\begin{abstract}
Two classes of ideals are introduced in a polynomial semiring $S[x]$, where $S$ is a commutative semiring with an identity. A structure theorem is given for each class.
\end{abstract}

1. Introduction. While much is known about ideals in rings and polynomial rings, little is known about ideals in polynomial semirings. In this paper, two classes of ideals in a polynomial semiring will be explored and a structure theorem for each class will be presented.

2. Fundamentals. There are different definitions of a semiring appearing in the literature. However, the definition used in [1] will be used throughout this paper. This definition is given as follows:

2.1. Definition. A set $S$ together with two binary operations called addition $(+)$ and multiplication $(\cdot)$ will be called a semiring provided $(S,+)$ is an abelian semigroup with a zero, $(S, \cdot)$ is a semigroup, and multiplication distributes over addition from the left and from the right.

A semiring $S$ is said to be commutative if $(S, \cdot)$ is a commutative semigroup. A semiring $S$ is said to have an identity if there exists $1 \in S$ such that $1 \cdot x=x \cdot 1$ for each $x \in S$.

2.2. Definition. A semiring $S$ is said to be a strict semiring if $a \in S, b \in S$ and $a+b=0$ imply $a=b=0$.

The set of nonnegative integers under the usual operations of addition and multiplication is a strict semiring.

2.3. Definition. A subset $I$ of a semiring $S$ will be called an ideal in $S$ if $I$ is an additive subsemigroup of $(S,+), I S \subset I$ and $S I \subset I$.

2.4. Definition. An ideal $I$ in a semiring $S$ will be called a $k$-ideal if $a \in I, b \in S$ and $a+b \in I$ imply $b \in I$.

2.5. Definition. An ideal $M$ in $S[x]$, where $S$ is a commutative semiring with an identity, will be called monic if $\sum a_{i} x^{i} \in M$ implies $a_{i} x^{i} \in M$ for each $i \in\{0,1,2, \ldots, n\}$.

2.6. Definition. An ideal $F$ in $S[x]$, where $S$ is a commutative semiring with an identity, will be called monic free if $M$ is a monic ideal such that $M \subset F$

Presented to the Society, March 20, 1975 under the title Monic free ideals in a polynomial semiring; received by the editors September 23, 1974 and, in revised form, January 7, 1975.

AMS (MOS) subject classifications (1970). Primary16-02, 16A66; Secondary 16A78.

Key words and phrases. Strict semiring, monic ideals, monic free ideals, $k$-ideals, coefficient ideals, monic closure, mixed ideals. 
then $M=\{0\}$. Ideals which are neither monic nor monic free will be called mixed.

2.7. ExAmpLEs. Let $Z$ be the integers and $Z[x]$ the ring of polynomials over $Z$. Let $a x^{i} \in Z[x]$ where $i>0$ and $a \neq 0$. Then, clearly $\left(a x^{i}\right)$ is a monic ideal in $Z[x]$. Now let $F=(x+1)$ be the ideal in $Z[x]$ generated by $x+1$. Then $F$ is a monic free ideal in $Z[x]$. To see this, let $M$ be a monic ideal such that $M \subset F$. If $M \neq 0$, then $M$ being monic assures the existence of an element $a x^{i} \in M$ such that $a \neq 0$ and $i>0$. Without loss of generality, we may assume that $i$ is even. Thus, $a x^{i} \in F$, whence $a x^{i}=g(x)(x+1)$ for some polynomial $g(x)$ in $Z[x]$. Replacing $x$ by -1 yields $a=0$, a contradiction. Hence $M=0$ and $F$ is monic free.

Throughout this paper, unless otherwise stated, $S$ will be a commutative semiring with an identity and $S[x]$ will be the semiring of polynomials over $S$ in the indeterminate $x$.

3. Monic ideals. Let $\left\{I_{n}\right\}$ be an ascending chain of ideals in a semiring $S$ and $I^{*}=\left\{\sum a_{i} x^{i} \in S[x] \mid a_{i} \in I_{i}\right\}$.

3.1. TheOREM. $I^{*}$ is a monic ideal in $S[x]$.

Proof. Let $f=a_{n} x^{n}+\cdots+a_{0} \in I^{*}, g=b_{m} x^{m}+\cdots+b_{0} \in I^{*}$, and $h=c_{k} x^{k}+\cdots+c_{0} \in S[x]$. It follows from $\left(a_{i}+b_{i}\right) \in I_{i}$ that $f+g \in I^{*}$. Consider the product $h f=\sum p_{t} x^{t}$, where $p_{t}=\sum c_{i} a_{j}$ for $i+j=t$. Clearly $j \leqslant t$ and consequently, $a_{j} \in I_{t}$ since $\left\{I_{n}\right\}$ is an ascending chain. Hence $p_{t}=\sum c_{i} a_{j} \in I_{t}$ and $h f \in I^{*}$. Since $f \in I^{*}$, it follows that $a_{i} \in I_{i}$ and consequently $a_{i} x^{i} \in I^{*}$.

At this point the following question may be asked: Does every monic ideal in $S[x]$ come from an ascending chain of ideals in $S$ ? To answer this question, a method of constructing an ascending chain of ideals in $S$ from a given ideal in $S[x]$ is needed.

Let $A$ be an ideal in $S[x]$ and $A_{i}=\left\{a \in S \mid\right.$ there is an $f \in A$ such that $a x^{i}$ is a term of $f$ \}.

3.2. THEOREM. If $A$ is an ideal in $S[x]$, then $\left\{A_{n}\right\}$ is an ascending chain of ideals in $S$.

Proof. For $a \in A_{i}$ and $b \in A_{i}$ there are polynomials $f \in A$ and $g \in A$ such that $a x^{i}$ and $b x^{i}$ are terms of $f$ and $g$ respectively. Consequently, $f+g \in A,(a+b) x^{i}$ is a term of $f+g$ and $a+b \in A_{i}$. If $c \in S$, then $c f \in A$ and it follows that $c a x^{i}$ is a term of $c f$. Consequently $c a \in A_{i}$. Since $x f \in A$, it is clear that $a x^{i+1}$ is a term of $x f$ and $A_{i} \subset A_{i+1}$.

For an ideal $A$ in $S[x]$, the ascending chain of ideals $\left\{A_{n}\right\}$ in $S$ will be called coefficient ideals. Let $A^{*}=\left\{\sum a_{i} x^{i} \in S[x] \mid a_{i} \in A_{i}\right\}$. Then $A \subset A^{*}$ and Theorem 3.1 assures that $A^{*}$ is a monic ideal in $S[x]$.

3.3. TheOREM. An ideal $A$ in $S[x]$ is monic if and only if $A=A^{*}$.

Proof. Theorem 3.1 assures that $A$ is monic if $A=A^{*}$. Suppose $A$ is monic and $f=a_{n} x^{n}+\cdots+a_{0} \in A^{*}$. Then $a_{i} \in A_{i}$ and there is a polynomial $g_{i} \in A$ such that $a x^{i}$ is a term of $g_{i}$. $A$ being monic assures that $a_{i} x^{i} \in A$. 
Consequently $f \in A$ and $A^{*} \subset A$. From $A \subset A^{*}$ it follows that $A=A^{*}$.

This theorem assures that all monic ideals in $S[x]$ can be structured from ascending chains of ideals in $S$. What about monic $k$-ideals?

3.4. Theorem. A monic ideal $M$ in $S[x]$ is a $k$-ideal if and only if $M_{i}$ is a $k$ ideal in $S$ for each $i$.

Proof. Suppose $M$ is a monic $k$-ideal in $S[x]$. Then $M=M^{*}$. Let $a \in M_{i}, b \in S$ and $a+b \in M_{i}$. Then $a x^{i} \in M,(a+b) x^{i}=a x^{i}+b x^{i}$ $\in M$ and $b x^{i} \in M$ since $M$ is a $k$-ideal. Consequently $b \in M_{i}$ and $M_{i}$ is a $k$ ideal. Conversely, suppose each $M_{i}$ is a $k$-ideal, $f=a_{n} x^{n}+\cdots+a_{0} \in M, g$ $=b_{m} x^{m}+\cdots+b_{0} \in S[x]$ and $f+g \in M$. Then $a_{i} \in M_{i}, a_{i}+b_{i} \in M_{i}$ and it follows that $b_{i} \in M_{i}$. Consequently $b_{i} x^{i} \in M$ and $g \in M$. Therefore $M$ is a $k$-ideal.

3.5. Definition. For an ideal $A$ in $S[x]$, the set $\bar{A}=\cap\{M \mid M$ is monic and $A \subset M\}$ will be called the monic closure of $A$.

It is evident that for any ideal $A$ in $S[x], \bar{A}$ is a monic ideal and consequently $\bar{A}=A^{*}$. It is easy to show that if $A \subset B$, then $\bar{A} \subset \bar{B}$.

3.6. TheOREM. If $A$ and $B$ are ideals in $S[x]$, then $\bar{A} \cap \bar{B}=\overline{A \cap B}$.

Proof. $\bar{A} \cap \bar{B} \subset \overline{A \cap B}$ is clear. Let $f=\sum a_{i} x^{i} \in \overline{A \cap B}$. Since $\overline{A \cap B}$ is monic, $a_{i} x^{i} \in \overline{A \cap B}$. Thus there is a polynomial $g_{i} \in A \cap B$ such that $a_{i} x^{i}$ is a term of $g_{i}$. Now $g_{i} \in A$ and $g_{i} \in B$. Hence $a_{i} x^{i} \in \bar{A}$ and $a_{i} x^{i} \in \bar{B}$ and it follows that $a_{i} x^{i} \in \bar{A} \cap \bar{B}$. Consequently $f=\sum a_{i} x^{i} \in \bar{A} \cap \bar{B}$ and $\overline{A \cap B} \subset \bar{A} \cap \bar{B}$. Whence $\bar{A} \cap \bar{B}=\overline{A \cap B}$.

4. Monic free ideals. Of the monic free ideals in a polynomial semiring, the monic free $k$-ideals are the most interesting. The following lemma is important to the study of monic free $k$-ideals.

4.1. Lemma. If $A$ is a $k$-ideal in $S[x], f=a_{n} x^{n}+\cdots+a_{0} \in A$, and $\tau$ is a nonnegative integer, then

$$
\left(a_{n} x^{n}+\cdots+a_{i+1} x^{i+1}+a_{i-1} x^{i-1}+\cdots+a_{0}\right)^{2 \tau+1}+\left(a_{i} x^{i}\right)^{2 \tau+1} \in A .
$$

Proof. By induction on $\tau$. Let $f=h+g$ where $h=a_{n} x^{n}+\cdots+a_{i+1} x^{i+1}$ $+a_{i-1} x^{i-1}+\cdots+a_{0}$ and $g=a_{i} x^{i}$. Assume that $h^{2 \tau+1}+g^{2 \tau+1} \in A$. Since $A$ is an ideal it is clear that $\left[h^{2 \tau+2}+g^{2 \tau+2}\right] f \in A$ and $\left[h^{2 \tau+1}+g^{2 \tau+1}\right] h g \in A$. Then

$$
\begin{aligned}
{\left[h^{2 \tau+2}+g^{2 \tau+2}\right] f } & =\left[h^{2 \tau+2}+g^{2 \tau+2}\right](g+h) \\
& =h^{2 \tau+3}+g^{2 \tau+3}+\left[h^{2 \tau+1}+g^{2 \tau+1}\right] h .
\end{aligned}
$$

Consequently $h^{2 \tau+3}+g^{2 \tau+3} \in A$, since $A$ is a $k$-ideal, and $h^{2 \tau+1}+g^{2 \tau+1} \in A$ for all nonnegative integers $\tau$.

4.2. Theorem. Let $S$ be a strict semiring. A monic free ideal $F$ in $S[x]$ with a finite basis is not a $k$-ideal.

Proof. Suppose that the theorem is false and $F$ is a monic free $k$-ideal with 
a finite basis $B=\left\{g_{1}, g_{2}, \ldots, g_{n}\right\}$. Let $\Delta: S[x] \rightarrow Z^{+}$be a function defined as follows: (1) if $h=b_{n} x^{n}+b_{n-p} x^{n-p}+\cdots+b_{0}$ has degree $n$ and $b_{n-p} \neq 0$, then $\Delta(h)=p$, (2) if $h=a x^{n}, a \neq 0$, then $\Delta(h)=n$ and (3) if $h=0$, then $\Delta(h)=0$. Now let $\Delta\left(g_{i}\right)=c_{i}$. Since $F$ is monic free, $g_{i}$ contains at least two nonzero terms and consequently $c_{i} \geqslant 1$. Suppose $c=\max \left\{c_{1}, c_{2}, \ldots, c_{n}\right\}$ and consider $f=a_{n} x^{n}+a_{n-p} x^{n-p}+\cdots+a_{0} \in F$. Clearly $\Delta(f)=p$ and it follows from Lemma 4.1 that $f_{\tau}=\left(a_{n} x^{n}\right)^{2 \tau+1}+\left(a_{n-p} x^{n-p}+\cdots+a_{0}\right)^{2 \tau+1} \in F$ for each nonnegative integer $\tau$. Since $p$ is fixed and $\Delta\left(f_{\tau}\right)=(2 \tau+1) p$, the sequence $\{(2 \tau+1) p\}$ is an increasing sequence of integers. Consequently, there is a $\lambda$ such that $\Delta\left(f_{\lambda}\right)=(2 \lambda+1) p>c$. Also $f_{\lambda} \in F$ and $B$ a basis for $F$ assures that

$$
\text { (1) } f_{\lambda}=\left(a_{n} x^{n}\right)^{2 \lambda+1}+\left(a_{n-p} x^{n-p}+\cdots+a_{0}\right)^{2 \lambda+1}=h_{1} g_{1}+\cdots+h_{n} g_{n}
$$

for $h_{i} \in S[x]$. At least one of the products, say $h_{i} g_{i}$ must produce a term of degree $(2 \lambda+1) n$, since $\left(a_{n} x^{n}\right)^{2 \lambda+1}$ appears on the left side of (1). From $\Delta\left(g_{i}\right)=c_{i}$ it follows that $g_{i}=b_{m} x^{m}+b_{m-c_{i}} x^{m-c_{i}}+\cdots+b_{0}$. Moreover, $h_{i}$ must have a term of the form $d x^{(2 \lambda+1) n-m}$ and $d x^{(2 \lambda+1) n-m} g_{i}=d b_{m} x^{(2 \lambda+1) n}$ $+d b_{m-c_{i}} x^{(2 \lambda+1) n-c_{i}}+\cdots+d b_{0} x^{(2 \lambda+1) n-m}$ is part of the product $h_{i} g_{i}$. Since $S$ is a strict semiring, none of the terms in any of these products can vanish. Consequently the right side of (1) contains a term of degree $(2 \lambda+1) n-c_{i}$. A term of this degree is guaranteed because $g_{i}$ must contain at least two nonzero terms. Since $(2 \lambda+1) p>c \geqslant c_{i}$, it follows that

$$
\begin{aligned}
(2 \lambda+1) n & >(2 \lambda+1) n-c_{i} \geqslant(2 \lambda+1) n-c>(2 \lambda+1) n-(2 \lambda+1) p \\
& =(2 \lambda+1)(n-p) .
\end{aligned}
$$

The second highest term on the left side of $(1)$ is $(2 \lambda+1)(n-p)$. Hence a term of degree $(2 \lambda+1) n-c_{i}$ cannot appear on the left side of (1) because of (2), a contradiction.

4.3. CoRollary. Let $S$ be a strict semiring. If $F$ is a monic free $k$-ideal in $S[x]$, then every basis for $F$ is infinite.

The above results make it possible to prove the following structure theorem for monic free $k$-ideals in $S[x]$, where $S$ is a strict semiring.

4.4. THeOREM. Let $S$ be a strict semiring. If $F$ is a monic free $k$-ideal in $S[x]$, then $F=\cup F_{\alpha}$ where $\left\{F_{\alpha}\right\}$ is a proper ascending chain of ideals.

Proof. Corollary 4.3 assures that $F$ has an infinite basis, say, $B=\left\{g_{\alpha}\right\}$ for $\alpha \in A$. Well order the elements of $B$ and let $F_{0}=\left(g_{0}\right)$ and $F_{\alpha}=\sum_{\gamma<\alpha} F_{\gamma}$ $+\left(g_{\alpha}\right)$. It is easy to see that $\left\{F_{\alpha}\right\}$ is a proper ascending chain of ideals and $F=\cup F_{\alpha}$.

4.5. ExAmple. Consider the integers $Z$ and the nonnegative integers $Z^{+}$. Clearly $Z$ is a semiring and $Z^{+}$is a strict semiring. Define a mapping $\eta: Z^{+}[x] \rightarrow Z[i], i=\sqrt{-1}$, by $\eta(f(x))=f(i)$. It is clear that $\eta$ is a semiring homomorphism and that $F=\operatorname{ker} \eta$ is a $k$-ideal in $Z^{+}$. Let $M$ be a monic ideal such that $M \subset F$. If $a x^{t} \in M$, then $a x^{t} \in F$ and $\eta\left(a x^{t}\right)=a i^{t}=0$. Hence $a=0$ and it follows that $a x^{t}=0$. Consequently $M=0$ and $F$ is monic free. 
Now let $A_{0}=\left(x^{2}+1\right), A_{1}=\left(x^{6}+1\right)+A_{0}, \ldots, A_{n}=\left(x^{4 n+2}+1\right)+A_{n-1}$, $\ldots$, and let $A=\cup A_{i}$. Clearly $\left\{A_{i}\right\}$ is a proper ascending chain of ideals in $Z^{+}[x]$. If $f(x) \in A$, then there exists $p$ such that $f(x) \in A_{p}$ and it follows that $f(x)=\left(x^{2}+1\right) f_{0}(x)+\cdots+\left(x^{4 p+2}+1\right) f_{p}(x)$. Further,

$$
\begin{aligned}
\eta(f(x)) & =f(i)=\left(i^{2}+1\right) f_{0}(i)+\cdots+\left(i^{4 p+2}+1\right) f_{p}(i) \\
& =0 \cdot f_{0}(i)+\cdots+0 \cdot f_{p}(i)=0,
\end{aligned}
$$

and it follows that $f(x) \in F$. Consequently, $A \subset F$. Now suppose $f(x)$ $=a_{n} x^{n}+\cdots+a_{0} \in F$. Then $\eta(f(x))=f(i)=0$. Write $f(x)=f_{1}(x)$ $+f_{2}(x)$, where $f_{1}(x)$ has only odd degree terms and $f_{2}(x)$ has only even degree terms. It follows from $f(i)=0$ that $f_{1}(i)=0$ and $f_{2}(i)=0$. Using this and the fact that the coefficients of $f(x)$ are nonnegative integers, it is straightforward to show, by rearranging terms and factoring, that $f(x)=\left(x^{2}+1\right) g_{0}(x)$ $+\cdots+\left(x^{4 t+1}+1\right) g_{t}(x)$, where $g_{i}(x) \in Z^{+}[x]$. Hence $f(x) \in A$ and $F \subset A$. Consequently $A=F$. Thus $F$ is a monic free $k$-ideal with an infinite basis.

Next, let $N=(x+1)$ be the ideal in $Z^{+}[x]$ generated by $x+1$. It follows from Example 2.7 that $N$ is monic free in $Z^{+}[x]$. Thus $N$ is a monic free ideal with a finite basis. Now assume that $N$ is a $k$-ideal. It is clear that $(x+1)^{2}(x+1)=(x+1)^{3} \in N$ and $3 x(x+1) \in N$. Consequently,

$$
(x+1)^{3}=x^{3}+3 x^{2}+3 x+1=x^{3}+1+3 x(x+1)
$$

and $x^{3}+1 \in N$. But this gives $x^{3}+1=g(x)(x+1)$, for some $g(x) \in$ $Z^{+}[x]$, which is impossible. Thus $N$ is not a $k$-ideal.

5. Mixed ideals. When $E$ is a mixed ideal in $S[x]$ one can consider the monic part of $E$ and the monic free part of $E$. While $E$ may contain many monic ideals, it also contains a "largest" monic ideal.

5.1. Definition. When $E$ is a mixed ideal in $S[x]$ the set $E^{0}=\sum\left\{M_{\alpha} \mid M_{\alpha}\right.$ is monic and $\left.M_{\alpha} \subset E\right\}$ will be called the monic interior of $E$.

Obviously $E^{0}$ is a monic ideal in $S[x]$ and it follows from the definition of $E^{0}$ that $E^{0}$ is the maximal monic ideal contained in $E$. Also since $E$ is mixed, $E^{0} \neq E$.

5.2. Definition. When $E$ is a mixed ideal in $S[x]$ the set $b E=E-E^{0}$ will be called the boundary of $E$.

5.3. TheOREM. Let $S$ be a strict semiring. If $E$ is a mixed ideal in $S[x]$ then $E=E_{1} \cup E_{2}$ where $E_{1}$ is the maximal monic ideal contained in $E$ and $E_{2}$ is monic free.

Proof. Since $E_{1}=E^{0}$, it only remains to show that $E_{2}$ is monic free. Let $E_{2}=(b E)$ be the ideal generated by $b E$. To show that $E_{2}$ is monic free it is sufficient to show that the ideal $(b E)$ contains no nonzero elements of the form $a x^{i}$. Observe that the boundary $b E$ can contain no term of the form $a x^{i}$ since $\left(a x^{i}\right)$ would be a monic ideal contained in $E$ and $a x^{i} \in\left(a x^{i}\right) \subset E^{0}$. This is impossible since $b E \cap E^{0}=\varnothing$. Consequently, the ideal $(b E)$, being in a polynomial semiring, can contain no term of the form $a x^{i}$ since $b E$ is a basis for $(b E)$ and $S$ is a strict semiring. Thus $E_{2}$ is monic free. 
It is noted here that for a mixed ideal in $S[x]$ the ascending chain $E^{0} \subset E \subset \bar{E}$ is always proper.

\section{REFERENCE}

1. P. J. Allen, A fundamental theorem of homomorphisms for semirings, Proc. Amer. Math. Soc. 21 (1969), 412-416. MR 38 \#5856.

Department of Mathematics, University of Alabama in Birmingham, Birmingham, ALABama 35233 